\title{
Hour-timescale profile variations in the broad Balmer lines of the Seyfert galaxy Markarian 6
}

\author{
Norayr S. Asatrian \\ Byurakan Astrophysical Observatory, Armenia \\ email: asat@bao.sci.am
}

\begin{abstract}
Part of results of the multi-epoch intranight optical spectroscopic monitoring of the Markarian 6 nucleus carried out at the telescopes of 6-m of the Special Astrophysical Observatory (Russia), 2.6-m of the Byurakan Astrophysical Observatory (Armenia) and 2-m of the Tautenburg Observatory (Germany) is presented.

Observations were made in 1979, 1986, 1988-1991 and 2007-2009 during a total of 33 nights with an average sampling rate of 4 spectra per night. TV-scanner and long-slit spectrographs equipped with Image Tube and CCD detector arrays were used. Altogether we analyzed $110 \mathrm{H} \beta$ and $58 \mathrm{H \alpha}$ region spectra to search for intranight variability in the broad hydrogen emission line profiles. The typical spectral resolutions were $4 \AA$ for scanner spectra, $6 \AA$ for photographic spectra, and $5 \AA$ and $10 \AA$ for CCD spectra. The S/N ratio at the continuum level near the $H \beta$ and $H \alpha$ lines was in the range $15-50$.

The purpose of the search was to look for the characteristic variability signatures of different kinematical models of the broad emission-line region. We considered the centering and guiding errors which can result in differences between spectra.

We found variations in the broad Balmer line difference profiles on time scale of hour with the level of significance of $3.6 \sigma$ to $5.0 \sigma$. Variations take the form of narrow, small bumps located at the blue and red sides or only at the blue side of the lines. In the intermediate level of broad line flux, the $H \beta$ and $H \alpha$ profiles show fine structure. Detected profile changes occurred at the same radial velocity shifts as the details in the fine structure.

The variability is at least 2 orders of magnitude more rapid than any observed for broad Balmer line profiles in AGNs that we are aware of in the literature.

Discovered extremely rapid line-profile variability may be associated with reverberation effects. Two-sided profile changes may indicate the response of circularly rotating hydrogen clouds in the BLR to a light pulse from a central source. One-sided profile variations may be attributed to a response of a non-disk component: the subarcsec scale region of the jet.
\end{abstract}

Keywords. Galaxies, active, Markarian 6, spectroscopy, variability, profile

\section{Observations, data reduction and results}

In observations we used a small entrance aperture (comparable to the seeng disk). No correction for spectral sensitivity has been applied. To search for variations in the $H \beta$ and $H \alpha$ broad-line profiles we have examined the difference spectra within each night of observation. Before the spectra can be compared, they have been relatively scaled to the broad-line flux. The scaling algorithm of van Groningen \& Wanders (1992) was used. This method does not require fitting the continuum and provides higher precision of calibration.

Examples of $H \beta$ and $H \alpha$ broad-line profile fine structure and observed rapid profile variations are displayed in Figures 1-2. 


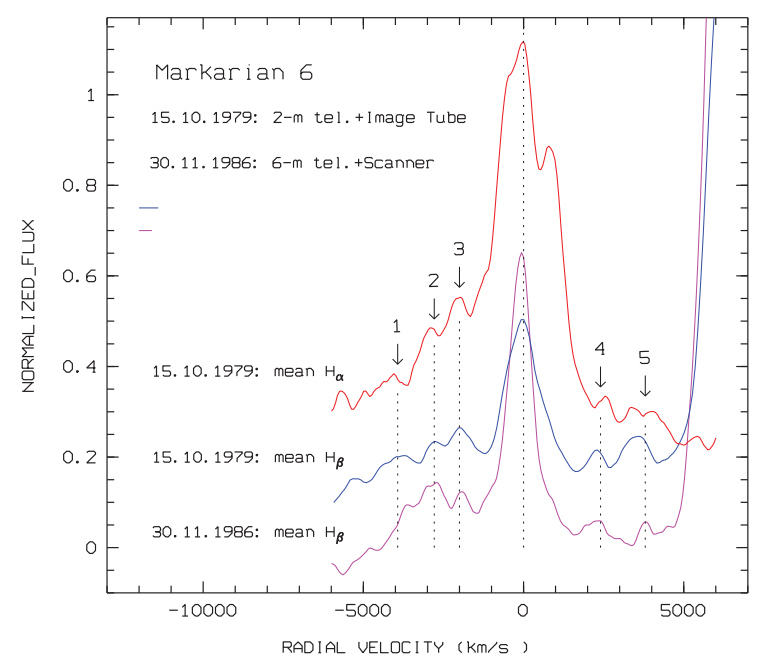

Figure 1. Comparison of the broad $H \beta$ and $H \alpha$ lines (observed in 7 years interval) showing example of steady fine structure of line profiles. It consists of the five narrow emission details (marked by arrows) located at the blue and red sides of the lines. Spectra of 1979 were obtained in the Tautenburg Observatory by P. Notni.

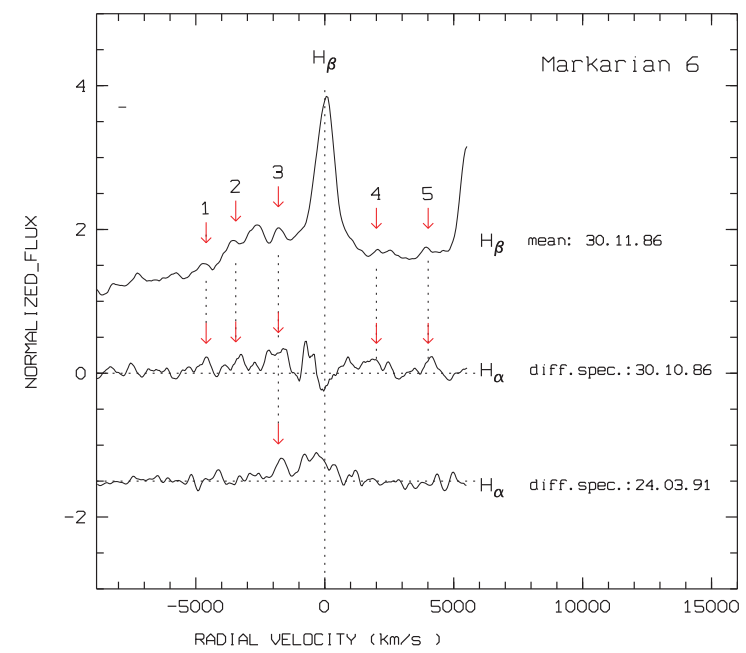

Figure 2. Two examples of the difference $H \alpha$ broad-line profiles illustrating the occurence of the hour-timescale profile changes at the same radial velocity shifts as the details in the $H \beta$ broad-line fine structure (marked by arrows). The parent spectra of these difference profiles can be found in Asatrian et al. 1999. They have been obtained at the 2.6-m telescope (in 1986) and at the 6-m telescope (in 1991). The apparent high residuals at the positions of the strong $H \alpha$ and $[\mathrm{NII}]$ narrow lines in the difference spectra are consequences of the TV scanner saturation and the centering and guiding errors in our observations.

\section{References}

Asatrian, N. S., Khachikian, E. Ye., \& Notni, P. 1999, in: Y. Terzian, D. W. Weedman \& E. Ye. Khachikian (eds.), Activity in galaxies and related phenomena (San Francisco: ASP), p. 409

van Groningen, E. \& Wanders, I. 1992, PASP, 104, 700 